\title{
Vertebrometría y discometría del segmento funcional vertebral cervical. Estudio comparativo y de concordancia entre Rx y TC
}

\author{
Alejandro González-Rebatu y González ${ }^{1 *}$, Joseph A. Lacayo-Simon², Lucienne Y. Lacayo-Simon ${ }^{3}$ \\ y Alicia M. Fierro-López ${ }^{4}$
}

${ }^{1}$ Servicio de Ortopedia, Hospital Regional 1. ${ }^{\circ}$ de Octubre, Instituto de Seguridad y Servicios Sociales de los Trabajadores del Estado, Ciudad de México; 2Servicio De Ortopedia, Hospital Regional de Puebla, Instituto de Seguridad y Servicios Sociales de los Trabajadores del Estado, Puebla; ${ }^{3}$ Práctica privada, Puebla; ${ }^{4}$ Práctica privada, Xpujil, Campeche. México

\begin{abstract}
Resumen
Introducción: La vertebrometría es útil para cirujanos que realizan reconstrucciones cervicales anteriores con fijación de placa, disectomía, instrumentación cervical. Objetivo: Evaluar la concordancia de la vertebrometría y discometría de los cuerpos vertebrales C4, C5 y C6 y el tamaño del disco intervertebral C4-C5, C5-C6 entre la radiografía lateral simple y la tomografía computarizada (TC). Material y métodos: Se realiza estudio de validación entre las medidas resultantes entre la proyección radiográfica lateral de columna cervical y la reconstrucción sagital de la columna cervical de la misma persona. Se captan 10 voluntarios sanos y se toman radiografías simples laterales de columna cervical y TC de columna cervical, se realizan mediciones del cuerpo vertebral C4, C5, C6 y el espacio intersomático C4-C5, C5-C6, se elaboran tablas y gráficas. Resultados: En proyección lateral simple de columna cervical y reconstrucción sagital en una TC simple con el método de Bland-Altman se realizó la comparación entre las medias de los dos estudios diferentes, donde se encuentra que la concordancia es similar. Conclusiones: Se encontró adecuada concordancia entre las medidas de las estructuras vertebrales cervicales en proyección radiográfica simple de la columna cervical y la reconstrucción sagital del mismo segmento.
\end{abstract}

Palabras clave: Cervicalgia. Vertebrometría. Discometría. Concordancia. Bland-Altman.

\section{Vertebrometry and dyscometry of the vertebral functional segment cervical. Comparative and concordance study between Rx and CT}

\begin{abstract}
Background: Vertebrometry is useful for surgeons who perform anterior cervical reconstructions with plate fixation, dissectomy, cervical instrumentation. To assess the concordance of vertebrometry and discometry of vertebral bodies C4, C5, C6 and the size of the intervertebral disc C4-C5, C5-C6 between plain lateral radiography and CT. (Computed Axial Tomography). Material and methods: A validation study was carried out between the resulting measurements between the lateral projection of the cervical spine and the sagittal reconstruction of the cervical spine of the same person. 10 healthy volunteers were taken and lateral plain radiographs of the cervical spine and CT of the cervical spine were taken. measurements of the vertebral body C4, C5, C6 and the interbody space C4-C5, C5-C6. Results: In a simple lateral view of the cervical spine and sagittal reconstruction in a simple CT with the Bland-Altman method, a comparison was made between the means of the two different studies, where the concordance was found to be similar. Conclusions: An adequate agreement was found between the measurements of the cervical vertebral structures both in simple radiographic projection of the cervical spine and sagittal reconstruction of the same segment.
\end{abstract}

Key words: Cervicalgia. Vertebrometry. Dyscometry. Concordance. Bland-Altman.

\footnotetext{
Correspondencia:

*Alejandro González-Rebatu y González

E-mail: alexrebattu@hotmail.com

Fecha de recepción: 09-06-2020

Fecha de aceptación: 01-03-2021

DOI: 10.24875/REMQ.20000017

Disponible en internet: 04-06-2021

Rev Esp Méd Quir. 2020;25:21-6

www.remq-issste.com

1665-7330 / @ 2021 Revista de Especialidades Médico-Quirúrgicas. Publicado por Permanyer México SA de CV. Este es un artículo Open Access bajo la licencia CC BY-NC-ND (http://creativecommons.org/licenses/by-nc-nd/4.0/).
} 


\section{Introducción}

El tratamiento quirúrgico de la enfermedad discal degenerativa tiene como objetivo reestablecer la altura del espacio discal y la liberación de las estructuras neurológicas involucradas; y como en cualquier tratamiento quirúrgico en ortopedia, el éxito o fracaso de la cirugía cervical consiste en la posibilidad de realizar una adecuada planeación de cada evento en particular. Hablando de la cirugía de estabilización cervical con fusión intersomática, conocer la altura ideal del espacio discal es indispensable para una fusión intersomática exitosa.

El tamaño del espacio discal en el segmento vertebral ha sido descrito por algunos autores. Se ha estudiado y definido la manera de medir el espacio discal, sin embargo, no se ha descrito la manera de realizar el cálculo del tamaño ideal del espacio discal en los pacientes con patología discal cervical degenerativa.

No existe trabajo similar para el cálculo del tamaño ideal del espacio discal, que se base en la medición del cuerpo vertebral (vertebrometría-discometría) ${ }^{1}$ del segmento vertebral funcional (vértebra-disco-vértebra) en el segmento cervical C4-C5-C6. Para validar dicha medición realizamos estudio de tomografía computarizada (TC) simple midiendo los cuerpos vertebrales y el espacio interdiscal C4-C5 y C5-C6.

Los cambios degenerativos tienen distinta expresividad en las diferentes estructuras. En algunas personas lo predominante es discal, con un pinzamiento y corona osteofítica y en otras alteraciones uncovertebrales $^{2}$.

La incidencia de conducto cervical estrecho en la población general se reporta entre 1.07-3.5 casos por cada 1,000 habitantes y una prevalencia de 3.5 por 1,000 habitantes, con un pico de incidencia en la sexta década de la vida ${ }^{3}$.

El dolor cervical es lo que se presenta más frecuentemente. En este intervienen principalmente factores musculares y ligamentarios relacionados con una postura inadecuada y fatiga muscular. El antecedente de una lesión cervical previa ha resultado ser un factor de riesgo adicional ${ }^{2,3}$.

Tanto para la patología de origen por degeneración discal o de estructuras óseas y/o ligamentarias existen mediadores neurogénicos del dolor liberados de los cuerpos neuronales y no neurogénicos liberados del disco y pueden tener un papel en la iniciación y perpetuación de la respuesta inflamatoria. El ganglio de la raíz dorsal es fuente de dolor al ser exquisitamente sensible a la deformación ${ }^{3,4}$.

La vertebrometría es útil para cirujanos que realizan reconstrucciones cervicales anteriores utilizando fijación de la placa, disectomía, instrumentación cervical. El diámetro anteroposterior de los cuerpos vertebrales son un parámetro. La fijación de tornillos y la colocación de la caja Peek (poliéter éter cetona) ${ }^{5,6}$. Así mismo, la altura del disco intervertebral cervical C3-C4, C4-C5, C5-C6, C6-C7 corresponde a 2/5 partes del cuerpo cervical suprayacente ${ }^{7,8}$.

Para validar cuál es la concordancia entre ambos estudios utilizaremos la medida de Bland-Altman ${ }^{9}$, quienes plantearon una manera más apropiada para analizar la concordancia, la que posteriormente se denominaría «límites de concordancia». El fundamento era evaluar si la comparación de los métodos permitía que uno reemplazara al otro con la suficiente precisión. Para esto había que considerar dos aspectos clave: cuan bien los métodos concuerdan en promedio, y cuan bien concuerdan para los individuos. La concordancia promedio se evalúa comparando el promedio de las diferencias de las mediciones de los individuos.

\section{Material y métodos}

\section{Material}

Se usan los siguientes equipos: tomógrafo Philips Diamond Select Brillance 64 cortes, equipo de Rayos $x$, computadora y software PAC'S para visualizar y medir las proyecciones.

\section{Diseño y tipo de estudio}

Validación, observacional, prospectivo, exploratorio, transversal y comparativo.

\section{Métodos}

Posteriormente a la firma de consentimiento informado, se toman:

- Proyecciones laterales simples de columna cervical, paciente en bipedestación con inclinación del rayo $15-20^{\circ}$ con respecto a la mandíbula y a distancia de $1.5 \mathrm{~m}$ de distancia entre el chasis y el rayo.

- TC cervical en tomógrafo Philips Diamond Select Brillance 64 cortes, el voluntario en decúbito 


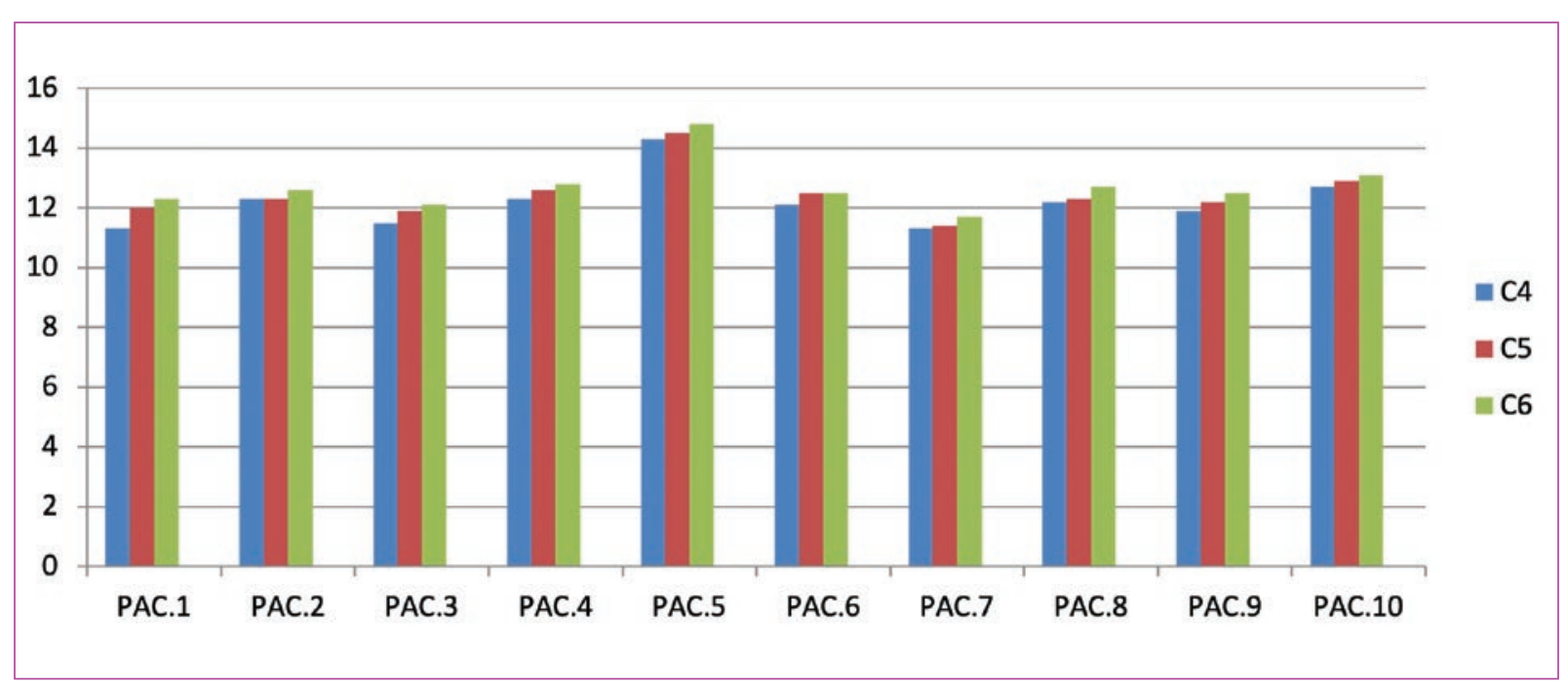

Figura 1. Altura de los cuerpos vertebrales.

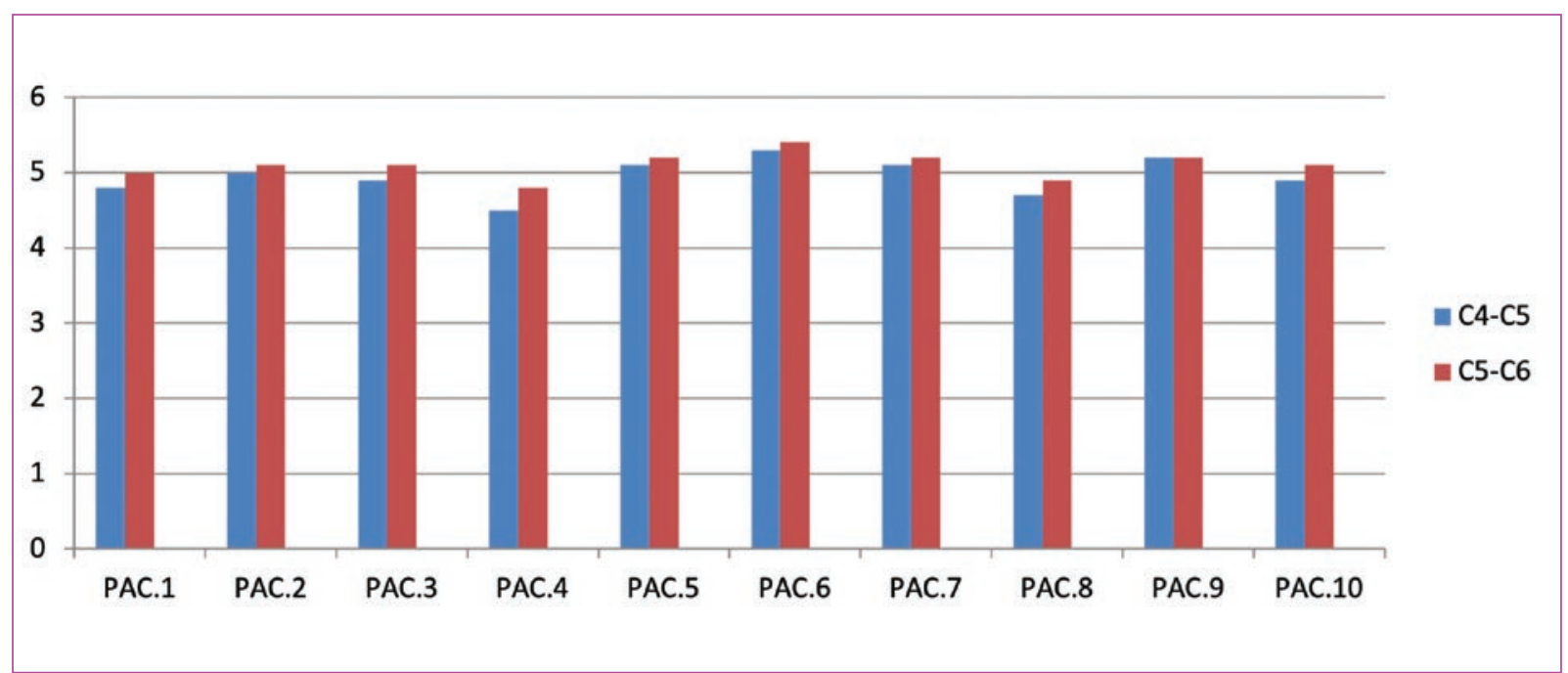

Figura 2. Altura de los discos C4-C5 y C5-C6.

supino, y se realizará reconstrucción sagital; posteriormente se realizará la medición de la altura de los cuerpos vertebrales C4, C5, C6 y el espacio intersomático C4-C5 y C5-C6 en los 10 voluntarios, se realizará la medición de los cuerpos vertebrales en la parte central de la plataforma superior a la plataforma inferior, en cada una de los cuerpos vertebrales, de la misma manera se medirá el espacio intervertebral de la parte central de la plataforma inferior de la vértebra superior a la plataforma superior de la vértebra inferior, que es lo que determina la altura del disco (Método de Farfan) ${ }^{10}$. Se recolectan los datos de: medida de altura de cuerpo vertebral $\mathrm{C} 4$, medida de altura de cuerpo vertebral C5, medida de altura de cuerpo vertebral C6, medida de espacio intervertebral de C4-C5, y C5-C6 en el software PAC'S tanto en proyección lateral radiográfica simple como en reconstrucción sagital en tomografía. Por método de medición Bland-Altman ${ }^{9}$ se graficará cada resultado para obtener la concordancia entre ambos estudios radiográficos.

Se realizan estudios a los 10 pacientes voluntarios, previo a su consentimiento informado, radiografías laterales verdaderas, donde se observen los cuerpos vertebrales de C4-CC5 y C6, y de la altura de los discos C4-C5 y C5-C6, en su parte central, se obtienen los resultados y se desglosan en la tabla 1 (Figs. 1 y 2). 
Tabla 1. Pacientes de radiografías

\begin{tabular}{|c|c|c|c|c|c|c|c|c|c|}
\hline Paciente & Edad & Estatura & Peso & IMC & $\begin{array}{l}\text { Altura del } \\
\text { cuerpo } \\
\text { vertebral C4 }\end{array}$ & $\begin{array}{l}\text { Altura del } \\
\text { cuerpo } \\
\text { vertebral C5 }\end{array}$ & $\begin{array}{l}\text { Altura del } \\
\text { cuerpo } \\
\text { vertebral C6 }\end{array}$ & $\begin{array}{c}\text { Espacio } \\
\text { intersomático } \\
\text { C4-C5 }\end{array}$ & $\begin{array}{c}\text { Espacio } \\
\text { intersomático } \\
\text { C5-C6 }\end{array}$ \\
\hline 1 & 24 & 1.65 & 70 & 25.7 & 11.3 & 12. & 12.3 & 4.8 & 5.0 \\
\hline 2 & 27 & 1.71 & 78 & 26.6 & 12.3 & 12.3 & 12.6 & 5.0 & 5.1 \\
\hline 3 & 24 & 1.68 & 74 & 26.2 & 11.5 & 11.9 & 12.1 & 4.9 & 5.1 \\
\hline 4 & 28 & 1.75 & 80 & 26.1 & 12.3 & 12.6 & 12.8 & 5.1 & 5.2 \\
\hline 5 & 26 & 1.80 & 88 & 27.1 & 14.31 & 14.56 & 14.86 & 5.3 & 5.4 \\
\hline 6 & 25 & 1.72 & 76 & 25.6 & 12.1 & 12.5 & 12.5 & 5.1 & 5.2 \\
\hline 7 & 29 & 1.60 & 65 & 25.3 & 11.3 & 11.4 & 11.7 & 4.7 & 4.9 \\
\hline 8 & 25 & 1.77 & 81 & 25.8 & 12.2 & 12.3 & 12.7 & 5.2 & 5.2 \\
\hline 9 & 26 & 1.72 & 75 & 25.3 & 11.9 & 12.2 & 12.5 & 4.9 & 5.1 \\
\hline \multirow[t]{2}{*}{10} & 28 & 1.76 & 80 & 25.8 & 12.7 & 12.9 & 13.1 & 5.2 & 5.4 \\
\hline & & & & & 110.6 & 127.16 & 127.16 & 50.2 & 51.6 \\
\hline Media & & & & & 11.06 & 12.71 & 12.71 & 5.02 & 5.16 \\
\hline
\end{tabular}

IMC: índice de masa corporal.

\begin{tabular}{|l|c|c|}
\hline $\mathbf{C} 4+\mathbf{C 5}=$ & $\mathbf{C 5}+\mathbf{C 6}=$ & Resultado final \\
\hline $11.06+12.71=23.77$ & $12.71+12.71=25.4$ & $42+48=92$ \\
\hline $23.77 / 2=11.88$ & $25.4 / 2=12.71$ & Media $=46$ \\
\hline 11.8 & 12.71 & $100 \%$ \\
$100 \%$ & 5.16 & \\
\hline 5.02 & 48.5 & \\
42.5 & $40.5 / 100=0.46$ \\
\hline $42 / 100=0.42$ & & \\
\hline
\end{tabular}

Posteriormente se realizan estudios de tomografía axial computada, en los mismos pacientes de la columna cervical con reconstrucción lateral en donde se realizan las mediciones de los cuerpos con la misma técnica que en las radiografías, midiendo la altura de los cuerpos vertebrales de C4-C5-C6 en su parte central y en los discos de C4-C5 y C5-C6 en la parte central. Se obtienen los resultados y se desglosan en la tabla 2 (Figs. 1 y 2 )

Se realiza estudio de validación entre las medidas resultantes entre la proyección lateral de columna cervical (Fig. 3) y la reconstrucción sagital de la columna cervical de la misma persona (Fig. 4). Se captan 10 voluntarios sanos y se toman radiografías simples laterales de columna cervical, así como TC de columna cervical y se realizan mediciones del cuerpo vertebral C4, C5, C6 y el espacio intersomático C4-C5, C5-C6.

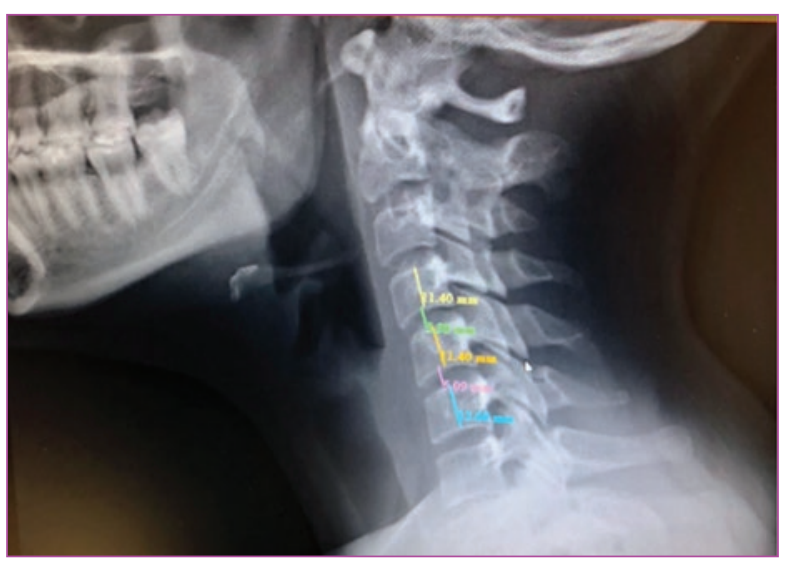

Figura 3. Radiografía simple con mediciones.

Se realiza la comparación entre las medidas de la proyección lateral radiográfica y la reconstrucción sagital. 
Tabla 2. Pacientes en tomografía computarizada

\begin{tabular}{|c|c|c|c|c|c|c|c|c|c|}
\hline Paciente & Edad & Estatura & Peso & IMC & $\begin{array}{l}\text { Altura del } \\
\text { cuerpo } \\
\text { vertebral C4 }\end{array}$ & $\begin{array}{l}\text { Altura del } \\
\text { cuerpo } \\
\text { vertebral C5 }\end{array}$ & $\begin{array}{l}\text { Altura del } \\
\text { cuerpo } \\
\text { vertebral C6 }\end{array}$ & $\begin{array}{c}\text { Espacio } \\
\text { intersomático } \\
\text { C4-C5 }\end{array}$ & $\begin{array}{c}\text { Espacio } \\
\text { intersomático } \\
\text { C5-C6 }\end{array}$ \\
\hline 1 & 24 & 1.65 & 70 & 25.7 & 11.3 & 12. & 12.3 & 4.8 & 5.0 \\
\hline 2 & 27 & 1.71 & 78 & 26.6 & 12.3 & 12.3 & 12.6 & 5.0 & 5.1 \\
\hline 3 & 24 & 1.68 & 74 & 26.2 & 11.5 & 11.9 & 12.1 & 4.9 & 5.1 \\
\hline 4 & 28 & 1.75 & 80 & 26.1 & 12.3 & 12.6 & 12.8 & 5.1 & 5.2 \\
\hline 5 & 26 & 1.80 & 88 & 27.1 & 14.31 & 14.56 & 14.86 & 5.3 & 5.4 \\
\hline 6 & 25 & 1.72 & 76 & 25.6 & 12.1 & 12.5 & 12.5 & 5.1 & 5.2 \\
\hline 7 & 29 & 1.60 & 65 & 25.3 & 11.3 & 11.4 & 11.7 & 4.7 & 4.9 \\
\hline 8 & 25 & 1.77 & 81 & 25.8 & 12.2 & 12.3 & 12.7 & 5.2 & 5.2 \\
\hline 9 & 26 & 1.72 & 75 & 25.3 & 11.9 & 12.2 & 12.5 & 4.9 & 5.1 \\
\hline \multirow[t]{2}{*}{10} & 28 & 1.76 & 80 & 25.8 & 12.7 & 12.9 & 13.1 & 5.2 & 5.4 \\
\hline & & & & & 107.6 & 124.6 & 127.16 & 50.2 & 51.6 \\
\hline Media & & & & & 10.76 & 12.46 & 12.71 & 5.02 & 5.16 \\
\hline
\end{tabular}

IMC: índice de masa corporal.

\begin{tabular}{|l|c|c|}
\hline $\mathbf{C 4}+\mathbf{C 5}=$ & $\mathbf{C 5}+\mathbf{C 6}=$ & Resultado final \\
\hline $10.76+12.46=23.22$ & $12.46+12.71=25.17$ & $43.2+41.2=84.4$ \\
\hline $23.22 / 2=11.61$ & $25.17 / 2=12.58$ & media $=46.2$ \\
\hline 11.6 & 12.5 & \\
$100 \%$ & $100 \%$ & 5.16 \\
\hline 5.02 & 46.2 & \\
\hline 44.2 & & \\
\hline
\end{tabular}

\section{Resultados}

Se realizan cálculos con los resultados, sacando la media de cada uno, C4-C5-C6, así como de los espacios discales C4-C5 y C5-C6. Una vez que se obtiene la media de los cuerpos y de los espacios discales se realiza una regla de tres para determinar el porcentaje de los espacios discales y de los cuerpos vertebrales.

Por lo tanto, obtenemos los siguientes resultados:

Rx: 46, TAC: 46.2

$46+46.2=92$

$92 / 2=4$

$46 / 100=0.46$

\section{Conclusiones}

Tras las mediciones y la realización de comprobaciones por reglas de tres podemos concluir que la

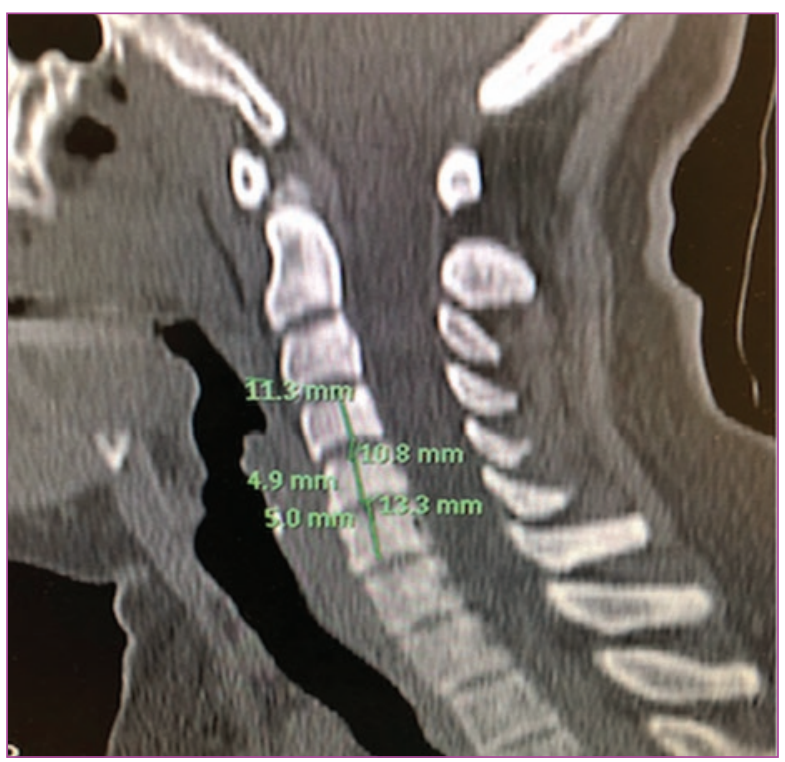

Figura 4. Reconstrucción tomográfica columna cervical y sus mediciones. 
altura discal corresponde al $46 \%$ de la altura vertebral en el segmento funcional cervical C4-C5 y C5-C6.

Se demuestra que la altura del disco no es equivalente a las $2 / 5$ partes del tamaño del cuerpo vertebral, si no que es un equivalente al $46 \%$.

Dándonos como resultado por medio de una comprobación de regla de tres, obtenemos una constante específica equivalente a 0.46

Por lo tanto, en pacientes con discartrosis, en los que se busca reestablecer la altura discal ideal, se toma como referencia la altura de los cuerpos vertebrales en milímetros (sacando la media) y se multiplica por 0.46 para obtener el tamaño ideal de caja intersomática que utilizar para una artrodesis. De esta forma se puede realizar una simple multiplicación en nuestra medición prequirúrgica en una radiografía simple para poder realizar la cirugía con la certeza de la altura de espacio discal que debe tener.

De esta forma no tenemos que estar adivinando la altura del espacio discal ni perdiendo el tiempo valorando cuál es la caja que mejor se puede adaptar durante la cirugía o la caja que más se aproxime a la medición que obtenemos.

Por lo tanto, consideramos que este artículo es único y original en su clase para columna cervical, por lo que es innovador, desarrollado por nosotros.

\section{Financiamiento}

Estudio realizado en el Hospital Regional $1^{\circ}$ de Octubre.

\section{Conflicto de intereses}

Los autores declaran que no existe ningún conflicto de intereses para la publicación del artículo.

\section{Responsabilidades éticas}

Protección de personas y animales. Los autores declaran que para esta investigación no se han realizado experimentos en seres humanos ni en animales.

Confidencialidad de los datos. Los autores declaran que han seguido los protocolos de su centro de trabajo sobre la publicación de datos de pacientes.

Derecho a la privacidad y consentimiento informado. Los autores han obtenido el consentimiento informado de los pacientes y/o sujetos referidos en el artículo.

\section{Bibliografía}

1. Vertebrometría y discometría del segmento funcional lumbar (técnica Rebatú-Murguía). González Rebatú y González A, Ortega Padrón R, Murguía Casas M, Vargas Burgos R, Vargas Lugo Salinas RB. Coluna/ Columna. 2017;16(2):112-5.

2. Silveri A, García Ascurra F. Estenosis raquídea cervical de origen artrósico. Actualización del tema y revisión de casuística. Rev Med Uruguay. 2000;16:221-31.

3. Alpízar-Aguirre A, Solano-Vargas JD, Zárate-Kalfopulus B, Rosales-Olivares LM, Sánchez-Bringas G, Reyes-Sánchez AA. Resultados funcionales de la cirugía del conducto cervical estrecho. Acta Ortop Mex. 2013;27(1):4-8.

4. Gálvez MM, Cordovez MJ, Okuma PC, Montoya MC, Asahi KT. Diagnóstico diferencial de hernia discal. Revista Chilena de Radiología. 2017;23(2):66-76.

5. Kanas M, Souassoni Leite M, Hisoshi R, del Curto D, Martins DE, Wajchenberg $\mathrm{M}$, et al. Comparación de los métodos de Farfan modificado y de Forbin para la medición de la altura del disco intervertebral. Coluna/Columna. 2014;13(1):31-4

6. Valle Ureta C, Valle Ochoa C, Rodríguez JC. Diámetro sagital del conducto raquídeo cervical en adultos ecuatorianos y su importancia en el tratamiento quirúrgico de la mielopatía espondilótica cervical [Internet]. Departamento de diagnóstico por imágenes, Hospital M.H. Alcívar; 2010. Disponible en: https://hospitalalcivar.com/uploads/pdf/DIAMETRO\%20 SAGITAL\%20DEL\%20CONDUCTO\%20RAQUIDEO\%20CERVICAL.pdf

7. Vargas-Mena R, Dufoo-Olvera M, García-López OF, López-Palacios JJ, Aburto-Trejo JA, Carranco-Toledo GA. Análisis morfométrico de los pedículos cervicales en una población mexicana. Acta Ortop Mex. 2011;25(6):366-71.

8. Bazaldúa Cruz JJ, González Larios A, Gómez Sánchez A, Villareal Silva EE, Velázquez Gauna EE, Sánchez Uresti A, et al. Morphometric study of cervical vertebrae C3-C7 in a population from Northeastern Mexico. Int J Morphol. 2011;29(2):325-30.

9. Kaur P, Stoltzfus J. Bland-Altman plot: A brief overview. Int J Acad Med. 2017;3(1):110-1.

10. Kanas M, Squassoni Leite M, Salvioni Ueta RH, Del Curto D, Martins $D E$, Wajchenberg $M$, et al. Comparación de los métodos de Farfan modificado e de Frobin para la medición de la altura del disco intervertebral. Coluna/Columna. 2014;13(1):31-4. 